\title{
Deformation measurement of internal components of ASDEX Upgrade using optical strain sensors
}

\author{
C. Vorpahl, W. Suttrop, M. Ebner, B. Streibl, H. Zohm and the ASDEX Upgrade Team \\ Max Planck Institute for Plasma Physics, EURATOM Association, Boltzmannstr. 2, 85748 Garching, Germany
}

\begin{abstract}
A fibre-optic measurement system to analyse the deformation of in-vessel components has successfully been developed, installed and commissioned at ASDEX Upgrade (AUG). This technology has thereby been qualified for in-vessel use at experimental fusion devices. AUG is equipped with an internal conductor for passive plasma stabilisation called the Passive Stabilisation Loop (PSL), on which the recently installed 16 internal coils (B-coils) are directly mounted. The PSL structure is highly prone to vibrations, and the risk of resonant oscillations in response to B-coil induced forces necessitated the development of the present diagnostic. The diagnostic system consists of 34 fibre-optic strain sensors incorporated in two glass fibres. It is completely insensitive to electromagnetic disturbances. The fibres are customised to avoid inconvenient excess fibre length in the vacuum vessel. They were tested for their neutron tolerance and vacuum compatibility prior to installation. The actual sensors are embedded in stainless steel carriers that were attached to the PSL, which is made of copper, by laser welding. Appropriate welding parameters were determined in view of the metallurgical dissimilarity. The weld quality was approved by tensile tests and microscopic investigations. Accurate in-vessel positioning of the sensors was assured using a 3D measurement system and coordinates from CAD. The data acquisition allows a sampling rate of $1 \mathrm{kHz}$. It was shown that the temporal and spatial resolutions of the system are sufficient to resolve the potentially dangerous bending eigenmodes of the PSL rings.
\end{abstract}

Keywords: ASDEX Upgrade tokamak, In-Vessel Coils, copper-steel laser welding, optical strain sensors (Fibre Bragg Gratings FBG)

\section{Introduction}

ASDEX Upgrade (AUG) is a mid-size tokamak fusion experiment. The vertical position control of the torus-shaped plasma is realised with a solid conductor inside the vacuum vessel called the Passive Stabilising Loop (PSL) as well as with active, feedback controlled vertical field coils [1]. The purpose of the passive component is to decelerate the motion of the plasma column to permit active control. This is achieved thanks to the high conductivity of the PSL and its proximity to the plasma. Plasma movements induce counteracting currents in the PSL. Figure 1 shows the geometry of the PSL (right) as well its position in the vessel (left). The PSL structure is composed of two solid copper rings (Cu-ETP), located at the outboard side above and below the mid-plane of AUG. They are interconnected by

Email address: christian.vorpahl@ipp.mpg.de ()

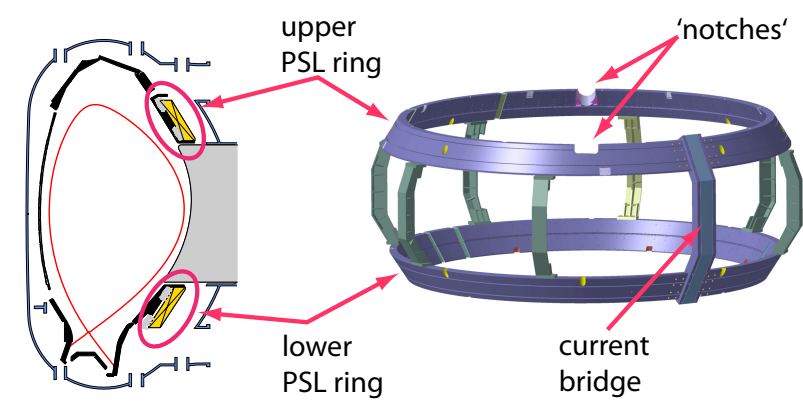

Figure 1: PSL position in the AUG vessel and geometry

8 equidistant bridges of which only one carries electric current and the other 7 are structural components. The assembly has a total mass of $8 \mathrm{t}$ and is suspended by the upper ring at 8 positions on hinges with ball-head type bearings, allowing radial excursions. Four tangential braces on each ring block the rotation around the 
vertical axis.

Currents flowing in the PSL can create Lorentz forces on the structure through interaction with the normal magnetic field component. These loads are strongest during plasma disruptions. Consequently, the initial PSL dimensioning was based on a worst-case disruption scenario. The recently installed 16 internal saddle coils or B-coils [2] are directly attached to the PSL rings [3]. Their operation results in $\mathrm{j} \times \mathrm{B}$-forces perpendicular to the PSL surface and proportional to the coil current. The B-coils have been operated in direct current at 1 $\mathrm{kA}$ during the last campaign and future plans include operation in alternating current up to $1 \mathrm{kA}$. This may cause resonant excitation not considered in the initial PSL dimensioning. Thus, an in situ measurement of its deformation was needed to investigate the new loading condition.

Optical strain gauges based on Fibre-Bragg-Gratings (FBGs) are used for this application. Despite the promising characteristics of this technology and plans to use FBGs in the context of the ITER test blanket [4] and vacuum vessel [5], it had not been tested within the vacuum vessel of a fusion device. An FBG is a type of Bragg reflector implemented into fibre optics which consists of a periodic variation in the refractivity of the fibre core. This causes light propagating in the fibre to be partially reflected at the grating location. The grating creates constructive interference of the reflected light at a particular wavelength characteristic of the grating's spacing [6]. The shift of this wavelength contains information about the strain of the grating along the fibre axis. Many gratings, each with its own unique characteristic wavelength, can be implemented in series on a single fibre. The different characteristic wavelengths allow spatial localisation of each grating. This considerably minimises cabling effort. As the information is contained in the reflection, the fibre only needs to be connected on one end, usually to a combined laser generator/spectrometer unit called FBG interrogator. Stress measurements rely on temperature measurements to decouple the strain components due to thermal expansion and due to stresses. Electromagnetic perturbations have no effect on this sensing technique. The extent of the grating on the fibre is of order $10 \mathrm{~mm}$ and is either fixed directly to the component of interest or embedded in a carrier to be fixed to the component.

On AUG, the steel carriers of the sensors needed to be fixed to the copper surface of the PSL. The severe dissimilarities of these materials required a weld quality assessment. One major risk is hot cracking (solidification cracking) of the weld due to the different melting temperatures and thermal expansion properties, ag- gravated by the diffusion of copper to grain boundaries [7]. Another risk lies in the formation of hard intermetallic phases of copper and iron or alloying elements, which is detrimental especially for the fatigue strength as these provide points of crack initiation. Tensile tests of welded specimen were performed to investigate the importance of hot cracking and to determine appropriate welding parameters. Microscopic investigations and hardness measurements were carried out to detect potential intermetallics. Laser beam welding was selected for its high achievable energy density, which results in a very localised heat affected zone and reduces the thermal stresses and the crack probability. The precision of this method also minimises the risk of damaging the fibre that passes next to the welding points.

\section{Sensor placement}

Two identical optical fibres with 17 strain gauges and 2 temperature sensors each were installed on AUG, as illustrated in figure 2. They are mounted on the inner
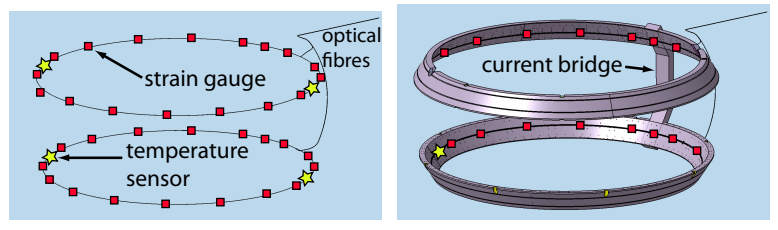

Figure 2: Sensing fibres (left) and fibre position on the PSL rings (right, support bridges not shown)

surfaces of the PSL, one fibre per ring. The strain sensors are positioned centrally with respect to the 16 vessel sectors, except for two on the current bridge. This placement covers the weak points of the PSL, namely the two opposite notches on the upper ring with drastically reduced cross-section (cf. fig. 1), the positions of the vertical hinges and various bores in both rings, which provide lines of sight for diagnostics. The two fibres revolve once around the torus and all four ends are led outside the vessel, providing redundancy. This means that one breakage per fibre can be tolerated without losing any sensor signal.

\section{Sensing material}

The sensor arrays are characterised essentially by the middle-wavelength of the gratings and the length of fibre between them. Two suppliers were assigned to produce the arrays according to a detailed specification. The first provided the fibres with engraved gratings. The second embedded the gratings into carriers made of stainless steel type 302 and calibrated the sensors according to industrial standards. The sensor positions and the path of the fibre in AUG were explicitly defined by CAD. This enabled a made-to-measure sensor array 
to be produced, avoiding any excess of fibre in the vessel. The sensors and the fibre were fixed on the front surface of the PSL rings, which is protected by wall tiles during operation. An existing groove on the PSL was primarily used to accommodate the fibre, minimising the necessary modification of tiles. The fibre is held in place by thin metal sheets covering the groove which are mounted at the locations of every tile gap. These sheets also shield the fibre from UV radiation from the plasma.

Commercial FBG interrogator units have a bandwidth of several tens of nanometres, the model employed at AUG is capable of detection in a window of $80 \mathrm{~nm}$. This window was divided into equal 'slots' for the strain sensors of one array. These slots determine the strain range that one sensor can detect, before its wavelength shifts into the neighbouring slot and the two adjacent sensors cannot be identified properly any more. The maximal strain values of the PSL were unknown, so the grating wavelengths were defined in ascending order along the fibre, skipping every second wavelength. When the highest value was reached in the middle of the fibre, the remaining wavelengths were assigned in decreasing order. This allows the fibre to be cut in the middle to double all slot widths if necessary while still being able to read out all sensors.

For vacuum compatibility, all critical parts of the sensor arrays were considered. The resin-drenched protective sleeve surrounding the glass fibre was replaced by one of pure silica glass fabric and the connectors' bend protection by PEEK parts. The adhesive used in minor quantities in the connectors and carriers is a hightemperature epoxy meeting low outgassing standard ASTM E595. The fibre is clad with a high-temperature polyimide free of halogens, which are particularly detrimental to the plasma. All parts, including the vacuum feedthrough passed an outgassing test at $150^{\circ} \mathrm{C}$ for 24 hours before installation. Regarding the neutron resistance, one sensor was seated on mid-plane level next to AUG during 100 discharges of the 2010 campaign. The fibre damping increase was below the detection limit of $0.1 \mathrm{~dB}$. The tolerable damping increase being $10 \mathrm{~dB}$, this yields a lifetime estimate of at least 10.000 discharges.

\section{Welding and Installation procedure}

Microscopy revealed that the intermixing zone formed in the weld does not exhibit microporosities as shown in fig. 3. Domains with high content of either copper or steel are clearly visible, consistent with the low solubility of copper in iron and vice versa.

A sample Vickers hardness measurement over the weld, the heat affected zone and the base material was
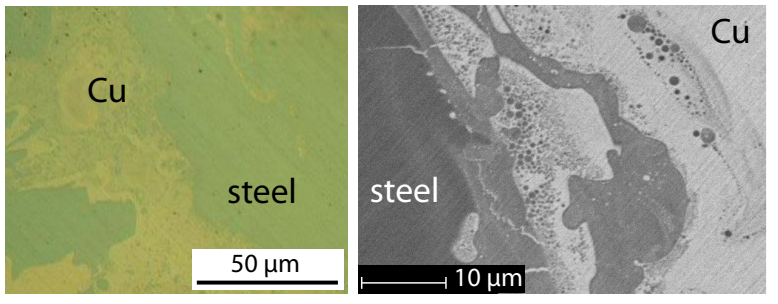

Figure 3: Intermixing zone of two test welds, optical microscopy (left), scanning electron microscopy (right)

realised, because hardness maxima in the weld would be a sign for intermetallics. It did not show local hardness peaks, as presented in fig. 4. Since the properties

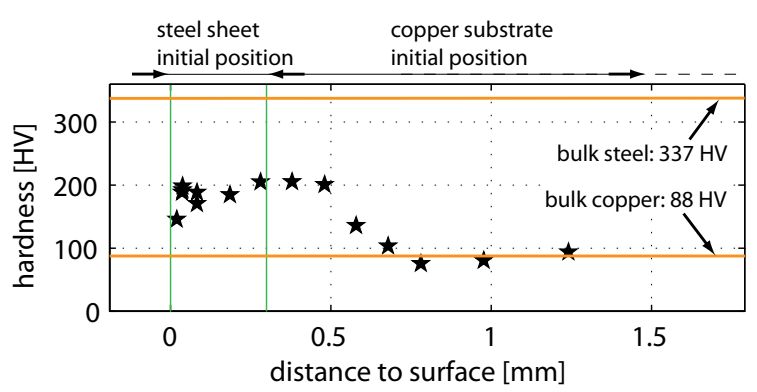

Figure 4: Hardness of test weld versus distance to surface

of the weld depend essentially on the process parameters a series of test welds with sample sheets and also spare carriers (cut in half, cf. fig. 5) was performed. Tensile tests were performed with both types of specimens. The carriers have 4 concave welding pockets with a material thickness of $0.3 \mathrm{~mm}$. All welds were realised aiming the beam at these pockets with the copper bulk material underneath. Seven laser pulses per pocket were applied with a beam focus diameter of $0.75 \mathrm{~mm}$ and a pulse frequency of $0.7 \mathrm{~Hz}$. Figure 5 shows a half-carrier specimen after welding before the tensile test. The pulse

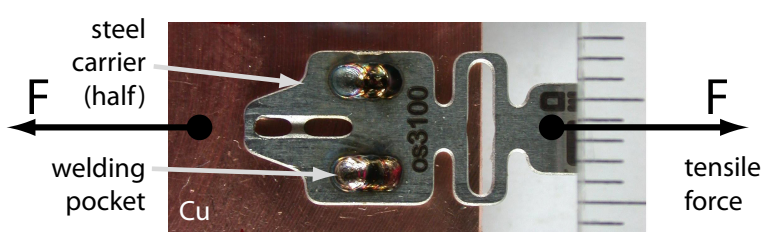

Figure 5: Half-carrier welding specimen (scale in $\mathrm{mm}$ )

energy is determined by the pulse length and the beam power, which is proportional to the laser flash lamp voltage. To find an appropriate working point in terms of these two parameters, the pulse energy was kept at constant values between 22-39 J while varying voltage and duration with respect to each other. Thus, a relevant portion of the parameter space was covered with reasonable effort. It showed that a compromise between deterioration of the steel surface by hot cracks and the penetration depth into the copper had to be found. This 
is also reflected in the tensile behaviour of the welds as depicted in fig. 6. There exist two different modes of

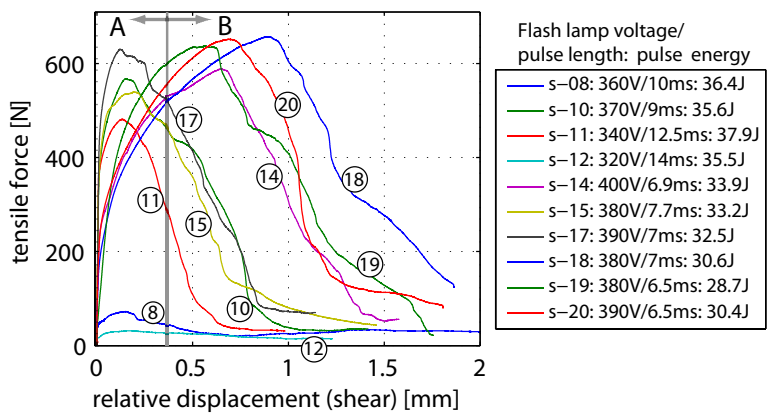

Figure 6: Tensile tests of copper-steel weld specimen

failure, substantially different in the amount of plastic deformation before reaching the force maximum. The first shows high stiffness at low shear (fig. 6, failure mode A, specimen 10,11,15,17) and low overall elongation. It corresponds to rupture at the interface of the copper matrix and the intermixing zone. The second exhibits considerable plastic deformation (mode B, specimen 14,18-20), and corresponds to failure of the carrier material (steel). Increasing the voltage, the superficial deterioration of the carrier increases, as well as the penetration depth into the copper. This means that failure in the carrier (mode B) gets more likely than failure in the copper (mode A). Domain A is preferable for welding the sensors because the high initial stiffness is beneficial for measurement precision. Therefore, a parameter set on the safe side to avoid excessive hot cracks on the steel surface was chosen. The lowest tensile strength of 8 spare carriers that were welded on solid copper using these parameters was $580 \mathrm{~N}$. A force-strain dependency test of a sensor carrier yielded a force of $21.4 \mathrm{~N}$ at the maximum design strain of the sensors, which is $2,500 \mu \epsilon$. Hence, the safety factor amounts to 27 , which satisfies the present application even under fatigue conditions.

The used pulsed Nd:YAG laser welder permits the separation of the welding head from the laser generator which remained outside the vacuum vessel. The head, supplied by a cable harness for remote control, laser transmission and shielding gas supply was mounted inside the vessel on a versatile holding device. Figure 7 illustrates the setup. The sensor positions, predefined by $\mathrm{CAD}$, were located with a 3D measurement arm with an accuracy of $0.5 \mathrm{~mm}$. In addition to the mere strength of the weld joints discussed earlier, comparability of the sensor signals requires identical stiffness which calls for a repeatable weld gap. Therefore, a clamping device was designed for applying a well-defined, constant nor-

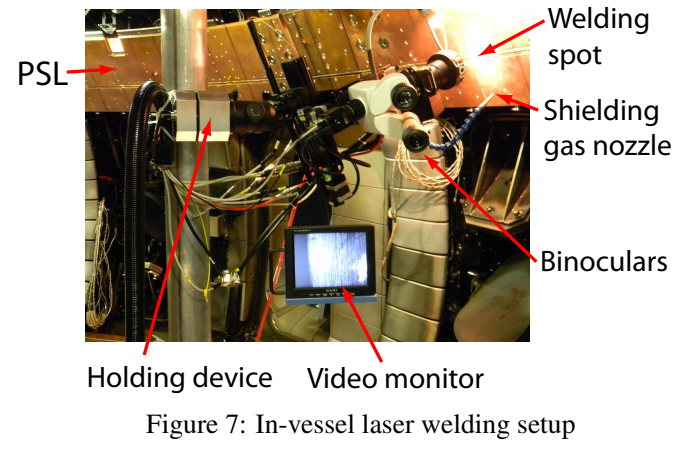

mal pressure on the sensor carrier during welding. This tool is equipped with a suction-cup and a bayonet-lock mechanism which allows the welding to be performed by a single person.

\section{Results and Discussion}

The measurement is implemented as a standard AUG diagnostic. It is sampled at $1 \mathrm{kHz}$, maintenance-free and runs automatically during every discharge. Figure 8 presents time traces of exemplary strain signals during two different scenarios: A plasma disruption (a) and excitation of the quadratic $(n=4)$ PSL eigenmode by AC operation of the B-coils at $110 \mathrm{~A}$ with toroidal field but no plasma (b). Figure 9 shows approximate PSL shapes

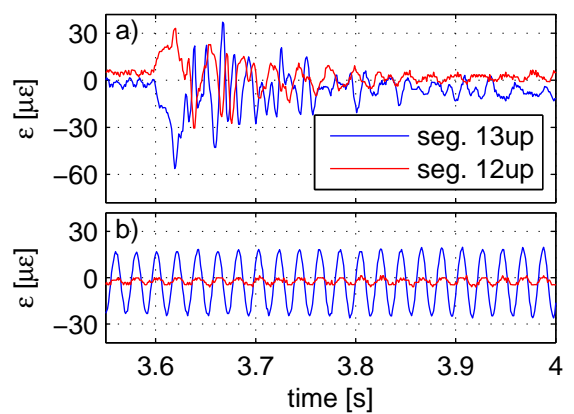

Figure 8: PSL upper ring strain signals of sector 12 and 13, disruption \#26026 (a) and AC B-coil operation \#27488 (b, n=4, I=110 A, excitation frequency sweep $45-60 \mathrm{~Hz}$, PSL resonance peak at $49.1 \mathrm{~Hz}$ )
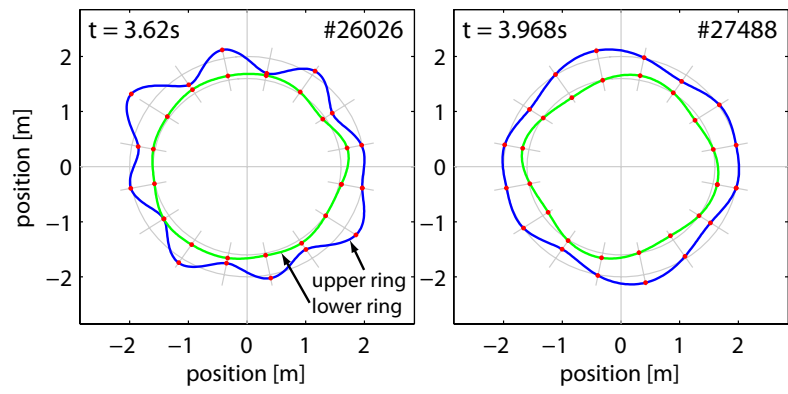

Figure 9: PSL top view prior to disruption \#26026 (left) and during AC B-coil operation test \#27488 (right, arbitrary scale)

(top-view) calculated using a cubic spline shape function, and assuming inverse linearity of measured local 
strain and deflection. The two graphs are snap-shots of both discharges illustrated in figure 8 . The deflections are in the sub-millimeter range and were exaggerated for visibility. It has shown that even the deformation of the PSL due to minor vertical plasma shifts and vertical field coil activities can be detected.

[1] V. Mertens, G. Raupp, W. Treutterer, Fusion Sci. Technol. 44 (2003) 593-604.

[2] W. Suttrop, et al., Fusion Eng. Des. 84 (2009) 290 - 294.

[3] T. Vierle, et al., Fusion Eng. Des. 84 (2009) 1928 - 1932.

[4] A. Puma, et al., Fusion Eng. Des. 85 (2010) 1642-1652.

[5] K. Ioki, Fusion Eng. Des. 85 (2010) 1307-1313.

[6] K. Hill, G. Meltz, J. Lightwave Technol. 15 (1997) 1263 -1276.

[7] Z. Sun, J. Ion, J. Mater. Sci. 30 (1995) 4205-4214. 